\title{
Social and ethical checkpoints for bottom-up synthetic biology, or protocells
}

\author{
Mark A. Bedau $\cdot$ Emily C. Parke $\cdot$ Uwe Tangen • \\ Brigitte Hantsche-Tangen
}

Received: 11 May 2009/Revised: 2 July 2009/Accepted: 30 July 2009

(c) The Author(s) 2009. This article is published with open access at Springerlink.com

\begin{abstract}
An alternative to creating novel organisms through the traditional "top-down" approach to synthetic biology involves creating them from the "bottom up" by assembling them from non-living components; the products of this approach are called "protocells." In this paper we describe how bottom-up and top-down synthetic biology differ, review the current state of protocell research and development, and examine the unique ethical, social, and regulatory issues raised by bottom-up synthetic biology. Protocells have not yet been developed, but many expect this to happen within the next five to ten years. Accordingly, we identify six key checkpoints in protocell development at which particular attention should be given to
\end{abstract}

M. A. Bedau ( $\square)$

Reed College, Portland, USA

e-mail: mab@reed.edu

M. A. Bedau - E. C. Parke

ProtoLife Inc, San Francisco, USA

M. A. Bedau - U. Tangen

European Center for Living Technology, Venice, Italy

M. A. Bedau

European School of Molecular Medicine, Milan, Italy

M. A. Bedau

Initiative for Science, Society, and Policy, Copenhagen,

Denmark

E. C. Parke

University of Pennsylvania, Philadelphia, USA

U. Tangen

BioMIP, Ruhr-Universität Bochum, Bochum, Germany

U. Tangen $\cdot$ B. Hantsche-Tangen

Protostream GmbH, Dortmund, Germany specific ethical, social and regulatory issues concerning bottom-up synthetic biology, and make ten recommendations for responsible protocell science that are tied to the achievement of these checkpoints.

Keywords Protocells - Synthetic biology ·

Ethical issues $\cdot$ Social issues $\cdot$ Regulation

\section{Protocells, or bottom-up synthetic biology}

Synthetic biology is the attempt to "engineer complex artificial biological systems to investigate natural biological phenomena and for a variety of applications" (Andrianantoandro et al. 2006; see also Endy 2005; European Commission 2005; Serrano 2007; Purnick and Weiss 2009). Most of the best-known work in synthetic biology is "top down" in the sense that it starts with some pre-existing natural living system and then re-engineers it for some desired purpose (e.g., Martin et al. 2003; Ro et al. 2006; Levskaya et al. 2005), perhaps by synthesizing (Smith et al. 2003; Gibson et al. 2008) or transplanting (Lartigue et al. 2007) entire genomes. Another approach to engineering novel biological systems works strictly from the "bottom up" in the sense that it attempts to make new simple kinds of minimal chemical cellular life, using as raw ingredients only materials that were never alive. ${ }^{1}$ These bottom-up

\footnotetext{
${ }^{1}$ Unfortunately, the 'top-down' vs. 'bottom-up' terminology has been used in many different ways in the synthetic biology literature. In this paper we follow the terminological conventions of Rasmussen et al. (2009a), but a variety of alternative conventions exist. A superficial survey of the synthetic biology literature turned up at least five different distinctions that have been marked with the 'top-down' vs. 'bottom-up' terminology: (1) the distinction between building a minimal living cell by using extant nucleic acids and enzymes vs. synthesizing all components from very simple molecules under
} 
creations are often called "protocells" (Rasmussen et al. 2009a), as we explain below. In the long run, bottom-up and top-down synthetic biology will increasingly blend and become harder to distinguish. ${ }^{2}$ In the meantime, though, it is important to separate them, not least because their social and ethical profiles differ. The ethical, social, and regulatory challenges raised by top-down synthetic biology have received considerable attention over the past decade (Cho et al. 1999; Brent 2006; Maurer et al. 2006; Garfinkel et al. 2007; ETC Group 2007; Boldt and Müller 2008; Balmer and Martin 2008; Schmidt et al. 2009). Bottom-up synthetic biology has only just begun to receive similar scrutiny (Bedau and Parke 2009b).

In the present paper we outline the social, ethical, and regulatory issues that specifically involve bottom-up synthetic biology, or protocells. We identify six key milestones, or "checkpoints," in protocell science that should provoke special ethical, social, or regulatory scrutiny, and propose a series of recommendations for actions that these checkpoints should trigger. Fully autonomous protocells do not yet exist, but many expect that the first ones could exist in the laboratory within the next five to ten years and could survive in the natural environment outside of the laboratory within the next ten to twenty years. On that same timescale, we expect that protocells will also be ready for commercial applications and that they could become key components in a new generation of medical, industrial, and informational technologies. While the vast bulk of protocell work today is basic research, the driving force in the future will increasingly be private enterprise.

It is important to stress that the risks entailed by protocell research today are negligible-no higher than the everyday risks in typical undergraduate biology and chemistry laboratories. Yet the perception of risks from protocells today is not negligible, and protocells have virtually all of the features that tend to make perception of risk high (Cranor 2009). In addition, responsibly facing future risks involving protocells requires laying the proper

\footnotetext{
Footnote 1 continued

prebiotic conditions (Luisi 2002) (2) the distinction between in vivo and in vitro synthetic biology (European Commission 2005) (3) the distinction between learning about biological systems by traditional analytical decomposition of natural systems vs. synthetic biological construction of simplified artificial systems (Simpson 2006) (4) the distinction between constructing a minimal genome by removing inessential genetic material from natural genomes vs. adding desired functionality to simple natural organisms by re-engineering their genomes (Balmer and Martin 2008), and (5) the distinction between constructing a minimal cell by starting with a whole genome vs. starting with nucleotides (O'Malley et al. 2008).

${ }^{2}$ In addition to the top-down and bottom-up projects, synthetic biology also contains a research tradition that involves synthesizing biological systems in vitro (e.g., Kim et al. 2006; Szostak et al. 2001; Zhang et al. 2007; Lincoln and Joyce 2009).
}

foundation today. Thus, we think society should now start to face the unique ethical, social, and regulatory issues raised by bottom-up synthetic biology.

\section{The chemical nature of protocells}

In the protocell research community there is a near-consensus to characterize protocells as chemical systems with three interlocking chemical capacities or functions. Protocells are defined as self-assembling and self-reproducing chemical systems created through human artifice (but not merely by manipulating a natural living organism) that produce the following interlocking chemical properties: (1) spatial localization of components by containment (2) utilization of energy and raw materials from the environment by metabolism and (3) control of the containment and metabolism by chemical information that can be replicated and can mutate (see Box 1). The resulting chemical systems can reproduce themselves, and a population of them could adapt and evolve. ${ }^{3}$ This characterization of protocells captures the conception of minimal chemical cellular life that is generally (if not universally) accepted in the protocell research community (Rasmussen et al. 2009b). Thus, just as the engineering word 'prototype' refers to an artificial structure that represents the first simple working model of a designed system, the term 'protocell' refers to the first artificial chemical system that captures the minimal conditions of cellular forms of life.

When a protocell reproduces, the genetic information that controls its internal functioning might mutate. If different protocells contain different genetic information, the resulting behavioral differences could impact their ability to survive and reproduce. Thus, a population of protocells could undergo natural (or artificial) selection and in principle, over time, new kinds of protocells could arise in the evolutionary process. Error-free reproduction can be achieved in computer science applications; in chemistry, on the other hand, such reproduction is hardly achieved. Furthermore, it is undesirable because coping with changing environmental conditions requires the ability to adapt and evolve.

A chemical system that cannot autonomously reproduce itself is not a protocell. For example, what are called "artificial red blood cells"-inert microscopic chemical systems with the capacity to pick up, carry, and release oxygen in the blood stream (Shinji 2005; Orive et al. 2003) — do not meet this conception of a protocell. At best

\footnotetext{
3 Our use of the term 'protocell' follows that of Rasmussen et al. (2009a). We should note that the term also has somewhat different, though related, uses. See, e.g., Szostak et al. (2001) or O'Malley et al. (2008).
} 


\section{Box 1 Protocells}

Created through human artifice but not merely by manipulating a natural living organism (such as a bacterium), a protocell is a self-assembling and self-reproducing chemical system, with the following three properties:

1. It maintains its identity over time by spatially localizing its components in some form of container.

2. It utilizes free energy from its environment and digests environmental resources in order to maintain itself, grow, and ultimately reproduce. This use of energy and materials is a form of metabolism.

3. The containment and metabolism are under the control of replicable and inheritable chemical information that can be "mutated" when the protocell reproduces. This informational chemistry functions as a programmable genetic system.

The proper chemical integration of these three properties enables protocells to reproduce themselves, and a population of them could adapt and evolve by natural selection.

they could be called protocell precursors. It should be noted that protocells also do not include the "artificial cells" produced in top-down synthetic biology (recall discussion above), all of which involve subjecting existing life forms to more or less extreme forms of genetic or metabolic reengineering.

Since protocells are defined functionally, rather than materially, their chemistry might differ dramatically from that found in existing life forms (Rasmussen et al. 2009a). This is partly what makes the ethical and social profile of protocells distinctively different from top-down synthetic biology.

\section{Comparison with top-down synthetic biology}

Many of the ethical, social, and regulatory issues concerning protocells have analogs concerning top-down synthetic biology, but in some cases the bottom-up and top-down issues are different. The social risks from top-down synthetic biology prominently include laboratory security, bioterrorism (Brent 2006; Maurer et al. 2006; Garfinkel et al. 2007) and environmental issues (ETC Group 2007). Current protocell research typically requires no special laboratory security or risk management beyond that practiced in any high school or college biology or chemistry laboratory. This contrasts with top-down synthetic biology, which currently can engineer organisms that produce deadly toxins. At the same time, protocells are put together literally "from scratch," rather than just through redesign of existing living things. Humans have been modifying and redesigning living things since the beginning of civilization (e.g., through crop and animal husbandry); top-down synthetic biology can be viewed as the most recent form of this long tradition.
Creating the first bottom-up protocell, however, will mark the first time humans have synthesized life from wholly nonliving materials. When we do gain the ability to design and manipulate protocells to suit defined purposes, this could have profound impacts on our view of life, including human life and our picture of our own place in the universe. If this happens, the social impact of protocells could far outstrip that of top-down synthetic biology.

One other critical point of comparison between topdown and bottom-up synthetic biology concerns predictability and possible interaction with the environment. Much of synthetic biology embraces a vision of working with "BioBricks," which are "standard DNA parts that encode basic biological functions," and using them to "program living organisms in the same way that a computer scientist can program a computer" (BioBricks Foundation web site 2009). The aspiration of this vision of synthetic biology is to make the engineering of living systems as predictable and reliable as traditional engineering in information technology. By contrast, protocell research tends to emphasize the biochemical synergies and other unpredictable emergent properties found in even the simplest forms of life (Rasmussen et al. 2009b). These synergies and emergent properties mean that protocell engineering will hardly be as predictable and reliable as programming a computer. ${ }^{4}$

As synthetic biology progresses beyond semi-static or one-step processes and incorporates more dynamic control, it, too, will increasingly need to confront the ethical, social, and regulatory issues raised by unpredictability. The ability to chemically program protocells will make it much easier to design and construct them, and they will have much greater social and economic value. But the unpredictability of chemical programmability raises special concerns. Current programming paradigms and control theory both require full knowledge of the system of interest, which is usually assumed to be linear. We do not know today how to control nonlinear systems governed by unknown parameters, when the systems are as complex as the simplest natural cells (Rugh 1981/2002).

This makes programming protocells a significant challenge. Protocells are designed to be able to survive and reproduce in a changing environment. This requires that they detect and dynamically react to changes in their local neighborhood (e.g., changing nutrition sources). So, protocells are dynamic entities through which energy, resources, information, and waste flow, under the control of feedback loops. Part of the information that enables

\footnotetext{
${ }^{4}$ In protocell programming there is a tradeoff between predictability on the one hand, and adaptability and resilience on the other. Commercial applications will aim to strike the right balance between them.
} 
protocells to function properly is stored internally in a genome, perhaps constructed out of a nucleic acid like DNA, but this genome only partly controls the protocell. To program and control this sort of holistic dynamical system, including its embodied physical existence and its interactions with the environment, involves much more than controlling the genetic information inside it. The same holds for the genomes of natural life forms. Contrary to some of the hype accompanying the human genome project, knowing the entire human "book of life" (genome) has not automatically answered all the important questions about how to program human life (International Human Genome Sequencing Consortium 2001; Venter et al. 2001).

\section{Six checkpoints for protocell science and technology}

Because protocells do not yet exist, there is no need today for special measures or institutions to regulate them. However, it is useful and wise to begin thinking today about preparing for our future with protocells, and for the ethical, social, and regulatory issues they will generate (see Bedau and Parke 2009a, b).

Here we distinguish six key stages in protocell development that represent major steps toward their existence and eventual integration into our technology, and into our society in general. Of all the scientific milestones in protocell science and technology (Rasmussen et al. 2009a), these six "checkpoints" have special ethical, social, or regulatory implications:

Checkpoint A: Systematic and advancing research in protocell synthesis should trigger consideration of the attendant ethical, social and eventual regulatory implications. This checkpoint has already been reached, and discussion has already begun.

Checkpoint B: The technical feasibility of protocells is a major social and ethical checkpoint concerning protocells because it signals that all the main scientific hurdles to making autonomous protocells have been surmounted. This is the most critical checkpoint that precedes the actual existence of protocells.

Checkpoint C: Creating the first fully autonomous protocell in the laboratory would involve creating a selfassembling and self-reproducing chemical system with the properties of containment, metabolism, and programmability. This is the single scientific protocell achievement with the greatest social and ethical significance.

Checkpoint D: Protocells that could survive outside the laboratory have the potential to cause harm to human health and the environment, so the ability to survive in the environment should trigger a re-assessment of regulation and containment standards for protocells.
Checkpoint E: Actually releasing protocells outside the laboratory (possibly for commercial reasons) would have special social significance because protocells would be in direct contact with vastly many more forms of life, including people.

Checkpoint F: Protocells that are toxic or infectious would trigger the need for appropriate safety regulations. Toxic or infectious protocells that existed only in the laboratory (Checkpoint C) would already be a risk. That risk would dramatically multiply if protocells were used outside the laboratory (Checkpoint E) in medical or environmental applications, because their usefulness would hinge on their exponential proliferation.

There are a number of dependencies among the six checkpoints. Some checkpoints can arise only after others have already been met. For example, the technical feasibility of protocells (Checkpoint B) must precede the actual creation of the first protocells (Checkpoint $\mathrm{C}$ ), and the first creation of protocells (Checkpoint C) must precede their release in the environment (Checkpoint E). For a different kind of reason, actual release outside the laboratory (Checkpoint E) is a significant checkpoint only if protocells could survive outside the lab (Checkpoint D).

Note that the dependencies among the checkpoints do not follow strict alphabetic order. In particular, protocells could be toxic or infectious (Checkpoint F) before they could survive outside the lab (Checkpoint D) or are actually released in the environment (Checkpoint E), so Checkpoint $\mathrm{F}$ might arise before Checkpoints D and E.

The justifications for the checkpoints are varied. The mere existence today of systematic and advancing research on protocells (Checkpoint A) itself raises a number of ethical, social, and regulatory issues, although existing ethical, social, and regulatory practices and institutions might be adequate to address those issues. This will change, though, when protocells are within scientific reach and Checkpoint B is reached. Today the main remaining technical hurdle that is blocking the creation of protocells is closing the replication loop, that is, making a protocell that can autonomously reproduce itself along with all its functional components. This, in turn, requires reading internally-stored descriptions of the protocell's functional components and "translating" those descriptions into functional entities. All other chemical requirements for protocells either are already available or are relatively easy to foresee.

The technical feasibility of protocells (Checkpoint B) is an important checkpoint only because it opens the door to fully autonomous protocells (Checkpoint C). Checkpoint C has a pivotal position among our checkpoints: All previous checkpoints derive their importance only because they lead to the creation of protocells, and all subsequent 
checkpoints become significant only after protocells actually exist.

The first protocells will almost certainly be highly dependent on a very specific and carefully created laboratory environment. A further scientific achievement would be to enable them to survive outside of the laboratory (Checkpoint D). This is an important commercial goal. Once it is possible for protocells to survive in the external environment, a qualitatively different kind of risk arises and a series of new ecological questions become pressing: How quickly do protocells degrade in different environments? Do they degrade into something harmful? What side effects or collateral damage should be expected?

The actual release of protocells into the environment outside of the laboratory is Checkpoint E. The scale of protocell commercial application could mushroom and their numbers could skyrocket only after they exist outside the lab. When protocells leave the laboratory, they will either be intentionally released into the environment (initially in a highly targeted manner), or they will be confined in industrial facilities. At this juncture, environmental impact lessons concerning nanotechnology and top-down synthetic biology become applicable to protocells. Industrial use of protocells avoids certain social and regulatory concerns because the protocells are confined to industrial facilities and could be carefully monitored and controlled.

The precursors of autonomous protocells (which would exist before Checkpoint C) could be toxic or infectious, but the risks involved would greatly increase when the dangerous agents could reproduce exponentially, as fully autonomous protocells would. As noted above, toxic or infectious protocells (Checkpoint F) could exist before protocells could survive outside the lab (Checkpoint D) or are actually released in the environment (Checkpoint E). Toxic or infectious protocells would immediately become subject to existing tests and regulations for toxicity and infectiousness, but some of protocells' distinctive properties, such as their ability to adapt and evolve, might call for special measures. The severe new potential hazards presented by developments in nanotechnology and top-down synthetic biology hint at the challenges that will be raised by the existence of bottom-up protocells. There is considerable uncertainty about the exact scope and nature of those risks.

\section{Recommendations about protocells}

In this section we outline a series of recommendations for socially responsible and ethical protocell research and development. Each of the checkpoints described above raises a specific set of issues, so our recommendations are connected to the specific checkpoints that trigger them.
Advances in protocell research are already gaining public attention, and this attention will increase as work on protocells moves from the research to the development stage. Thoughtful communication with the public should be one of the earliest concerns on the agenda for socially and ethically responsible protocell research. As the technical feasibility of creating protocells (Checkpoint B) approaches, communications with the public should address the social and cultural concerns that arise regarding protocells (discussed below). It is also important in these early stages to avoid as much as possible the kind of media hype associated with, e.g., early cloning research and synthetic biology projects, and to stress to the public that the actual risk of protocell research today is negligible. The public will be more likely to accept this if they are supplied with clear information about the actual state of the science, rather than hype about prospects for protocells many years in the future. This leads to our first recommendation:

Recommendation 1 Success at reaching any major scientific milestone in protocell research, or any of the checkpoints identified above, should be clearly and promptly communicated to the public.

As seen already in top-down synthetic biology, the prospect of the ability to design and engineer entirely new life forms generates tension with a number of deep social, cultural, and religious norms and preconceptions (Cho et al. 1999). This prompts the recommendation that responsible stakeholders in protocell research should engage the public in discussing these concerns as early in the process of developing protocells as possible.

Recommendation 2 Reaching any of the checkpoints should be accompanied by open public discussion of social, cultural, religious, or other concerns and worries about protocells.

Social, cultural, and religious concerns may include (but are not limited to) the following kinds of issues:

- Conflict with religious doctrines. Many of the new technologies for manipulating life forms, such as in vitro fertilization, reproductive cloning, and embryonic stem cell research, seem to conflict with the doctrines of various religions (Kass 2002; Pullella 2008). These biotechnologies become controversial mainly when they are applied to human life, so protocell technology might escape similar condemnation for the foreseeable future. But since any new and powerful technology could indirectly impact human life, making protocells even in a research context might violate someone's deeply held religious doctrines.

- Violating nature. The "unnaturalness" of bottom-up protocells unsettles some people, because they feel that 
assembling life from scratch violates the sanctity or wisdom of life, or upsets the balance of nature (e.g., Fukuyama 2002). Admittedly, protocells might be quite unlike any currently existing form of life. However, the boundary between the natural and the artificial is difficult to draw, and protocells themselves have the potential to shift the boundaries of what would be considered natural. The ethical implications of these boundaries are controversial.

- Playing God. The worry here is not that only God could create wholly new forms of life, but that only a being with almost God-like understanding and wisdom would have the moral and scientific insight required to properly and judiciously exercise the capacity to create new forms of life (Bedau and Parke 2009a). Note that this concern does not depend on belief in any particular religious doctrine.

The ethical and cultural concerns that arise with respect to modifying life or creating new life carry a special weight when applied to bottom-up protocells. In particular, the concerns about violating nature and "playing God" mentioned above apply more significantly in the case of bottom-up protocells than they do in the case of top-down synthetic biology. Where to draw the line between the natural and the artificial is a complex matter, but the fact that protocells are made from nonliving materials intuitively makes them seem less natural (and thus, more objectionable, on this line of reasoning) than top-down synthetic biology organisms created by re-assembling preexisting biological parts. Similarly, if the playing God objection depends on how unnatural the new forms of life are, assembling life from the bottom up would seem to be more objectionable than re-engineering existing life.

Funding agencies significantly shape the course and conduct of research activities, and they can be an important force for change. Initiatives for funding protocell science should follow the lead of the Human Genome Project and set aside a fixed percentage of their funds for studies of the social, ethical, and legal implications of protocell development, and for public education on scientific advances in protocell science (Gaisser et al. 2008).

Recommendation 3 In order to carefully examine the social and ethical implications of protocells, once systematic and sustained protocell research is happening (Checkpoint A), funding for protocell research should set aside a small fraction (initially, 5\%) of total funds for the study of the ethical, legal, and social implications of protocell research and development.

In addition to the public education efforts mentioned above, an academic curriculum covering social and cultural aspects of protocell research will contribute to creating generations of responsible scientists in the field. A general culture of thoughtful and responsible action should be cultivated in the protocell research community. Forming a professional society (none exists so far) for bottom-up protocells will be a key step toward promoting such a culture.

Recommendation 4 Once systematic and sustained protocell research is happening (Checkpoint A), education in the benefits, risks, uncertainties, and best practices of protocell research should become a regular part of the synthetic biology research curriculum at colleges and universities.

Recently, there have been controversies in intellectual property law concerning new informational and biological technologies, such as whether and how algorithms and software should be patented, and whether patents should be granted for genetic material ranging from individual genes to whole genomes. This suggests that the special features of protocells will themselves undoubtedly raise new intellectual property challenges (Pottage 2009). One concern arises from the fact that protocells are part of a brand new, stillemerging technology. Because protocells are so new, existing intellectual property claims cover very little of the field and thus leave the field wide open for new claims. Some commentators are concerned that this will give first movers an opportunity to own and control extremely broad swaths of intellectual property; an example from top-down synthetic biology is expressed in the ETC Group's concerns (2007) about J. C. Venter's application to patent his modified forms of Mycoplasma genitalium (USPTO 2007).

Recommendation 5 By the time protocells are within technical reach (Checkpoint B), and at each subsequent checkpoint, intellectual property regulations should be reevaluated with regard to whether they adequately deal with the distinctive properties of protocells.

We expect unique issues to arise for bottom-up protocells in the area of intellectual property. The wide-open nature of the new field of bottom-up protocell technology-which makes it vulnerable to overly broad patents aimed at monopolies-will have serious legal implications. Furthermore, we expect strong (and possibly novel) ethical controversies will arise when it comes time to issue patents for bottom-up protocells. The social issues surrounding the patenting of life forms have been evident at least since the 1980 US Supreme Court case Diamond v. Chakrabarty (447 U.S. 303), which affirmed that genetically modified micro-organisms could be patented. One focal point of this debate is the legal issue of whether a genetically modified life form can really be called an "invention," since it is just a modification of a pre-existing biological entity that 
humans certainly did not create. The first living protocell created from nonliving material, however, will be unambiguously man-made; this will add a whole new dimension to the ethical debates around any claims of life forms as intellectual property.

The synthetic biology community has devoted attention to the threat that synthetic biology might be used by terrorists or other malevolent parties to create new bioweapons. It is conceivable that protocells could similarly be used as bioweapons, or as vehicles for espionage, or as tags for surreptitiously tracking people. This potential for dual use of protocells (that is, development for both peaceful and harmful aims) must be admitted, but it should not be overestimated or exaggerated. Protocells would be a poor choice for anyone intending to cause harm to people, for they would be difficult to control and target with precision. ${ }^{5}$ There are much easier ways to wreak havoc than using protocells.

Recommendation 6 Before protocells are technically feasible (Checkpoint B), the relatively low likelihood of malicious use of protocells should be clearly explained in communications concerning the risks of protocells. The potential for malicious use should be re-evaluated at each checkpoint once protocells become technically feasible (Checkpoint B).

As suggested earlier, it may be that existing oversight institutions provide some or even all of the oversight presently needed for protocells, given that they do not yet exist and are not yet within technical reach. Nevertheless, we should still review whether existing institutions provide adequate regulation for future protocell research and development, or whether the existence of protocells will reveal gaps in the current regulatory structures. This review process should begin now and should be completed before Checkpoint B (technical feasibility). As a part of this review, all existing institutions under whose oversight and regulation protocell development might fall should be equipped with the relevant knowledge to apply their mandates to this new technology. Oversight institutions should distinguish four different bodies that are subject to recommendations or regulations: (i) the scientific community engaged in protocell research (ii) the governmental agencies responsible for regulating protocell research and development (iii) companies that produce protocells commercially and (iv) consumer protection bodies.

\footnotetext{
5 It is telling that the most recent unclassified reference on unconventional warfare (Department of the Army 2008) devotes only one sentence in 248 pages to the possible danger of biological warfare. In principle, this area is already regulated by the Biological Weapons Convention.
}

Recommendation 7 Once the feasibility of autonomous protocells (Checkpoint B) is visible on the horizon, the special regulation issues raised by protocells must be reviewed. Proper oversight institutions for protocells should be established well before fully autonomous protocells exist (Checkpoint $\mathrm{C}$ ), and these institutions should be re-evaluated at each subsequent checkpoint.

A standardized system of classifying levels of precaution when handling biological agents exists, which uses a scheme of four biosafety levels. We recommend that some analogous classification system be developed for working with protocells in the laboratory. Certain features of protocells will merit special attention when implementing such a system. Some features can be predicted now, and some will be recognized only after protocells are capable of surviving outside the laboratory, and the creation of toxic or infectious protocells is a possibility (i.e., when Checkpoints $\mathrm{D}$ and $\mathrm{F}$ are reached). Features such as autonomous reproduction, adaptation, and evolution are novel and will be particular to protocells; we cannot assume that traditional biosafety levels developed without special attention to these powerful novelties will be adequate for their containment and regulation. Upon creation of the first fully autonomous protocell (Checkpoint C) and at every checkpoint thereafter, protocell safety classification should be reexamined and revised. This is because, by the time those checkpoints are reached, scientists will understand many more of the details connected with the actual safety issues and underlying mechanisms of protocells; these unknown details are the proper basis for resolving the details of this classification system.

Recommendation 8 Before autonomous protocells have been created (Checkpoint B), existing oversight institutions should develop both a classification of protocell safety levels and a list of best practices and protocols for safely using protocells in the laboratory.

Protocells will be able to metabolize material from their environments, reproduce, and evolve. Because they will be able to evolve, there is some chance that they could cause problems for human health or the environment. Therefore, as protocell research progresses, appropriate safety mechanisms should be implemented in anticipation of any such points where control could be lost (e.g., when protocells could potentially survive outside the laboratory, Checkpoint D). Society has had significant experience providing oversight and regulation with certain kinds of complex and potentially dangerous systems. For example, so-called "dependable" systems in computer science and engineering have resilient, built-in safeguards to prevent erroneous or dangerous behavior. Making protocells similarly "dependable" is a key goal. 
Recommendation $9 a$ When protocells are on the horizon (Checkpoint A) and before their technical feasibility is secured (Checkpoint B), a comprehensive plan for safety mechanisms should be developed.

Recommendation $9 b$ Before protocells have been created (Checkpoint $\mathrm{C}$ ), a comprehensive and thoroughly vetted set of safety mechanisms should be identified.

Recommendation 9c Before protocells could survive outside the laboratory (Checkpoint D), the vetted set of safety mechanisms should be implemented and thoroughly tested.

Recommendation 9d Before protocells are released outside the laboratory (Checkpoint E), thoroughly tested safety mechanisms and quality assurance measures should be deployed, and their success should be re-evaluated at subsequent checkpoints, so that the safety mechanisms can be adjusted and improved as necessary.

Recommendation 9e Before toxic or infectious protocells exist (Checkpoint F), a proper framework of regulations and procedures both for handling toxic and infectious agents and for providing the proper oversight of protocells must be implemented.

Once again, we hasten to point out that toxic or infectious protocells might exist (Checkpoint F) well before protocells could survive outside the laboratory (Checkpoint D) or are actually released in the environment (Checkpoint E). So, Recommendation 9e might be triggered before Recommendations 9c or 9d.

Recommendations 8 and 9 involve responsible regulation and risk management concerning protocells. Future developments in protocell technology will probably prompt the need for further actions on regulation and risk management beyond those listed here. Society must constantly reassess the responsible development of protocell technology once their autonomous existence becomes technically possible (Checkpoint B).

Until we approach actual release of protocells outside the laboratory (Checkpoint E), we will lack enough information to accurately evaluate the environmental impact of protocells. Protocells will be unlike existing life forms and they might impact the environment very differently, in ways that we will better understand only after we start to make the transition from research to development. We should start now to investigate the environmental impact of protocells in an anticipatory way, always remembering our current lack of full information and always accepting the responsibility to continue to re-evaluate the situation until protocells are actually released in the environment (Checkpoint E), and beyond. One issue that needs continual re-evaluation is the biocompatibility of protocells.
Protocells might directly interact with other organisms in the environment. In addition, the environmental impact of protocells might result from their impact on the abiotic environment (e.g., by increasing or decreasing greenhouse gases), which would indirectly affect forms of life that depend on that environment.

Recommendation 10 Oversight institutions should be mindful now of the potential future environmental impact of protocells. The possible and expected environmental impact of protocells should be openly discussed and investigated as soon as protocells become technically feasible (Checkpoint B), and oversight institutions should take concrete steps to evaluate and consider environmental impact as an explicit issue well before protocells could survive outside the laboratory (Checkpoint D).

Our recommendations about protocell risks and regulation are specifically tied to the unique features of bottom-up synthetic biology. Recommendation 8 about oversight institutions is contingent on the technical feasibility of protocells (Checkpoint B), but Checkpoint B is pending only for bottom-up, not top-down, synthetic biology. Recommendation 9 about safety mechanisms has a forward-looking five-part structure that mirrors Checkpoints B-F. These checkpoints are relevant only for bottom-up synthetic biology. It should be noted that the environmental impact of protocells (Recommendation 10) might be significantly less than that of top-down synthetic biology today, or at least significantly different, if protocells are made out of "unnatural" materials that interact minimally with their environment (i.e., materials that are different enough from those that make up current living organisms in some of the key ways described earlier in this paper).

The way in which each of the six checkpoints triggers specific recommendations is summarized in Table 1. Some recommendations concern checkpoints that trigger a discrete action (indicated with single check marks), such as communicating the latest scientific progress to the public (Recommendation 1) or re-evaluating regulations about intellectual property rights (Recommendation 5). Other recommendations involve creating ongoing processes (indicated with double check marks), such as creating and teaching a curriculum on the social and ethical issues concerning protocells (Recommendation 4) or implementing environmental controls (Recommendation 10). In general ongoing processes would continue through all subsequent checkpoints. Many recommendations first need to have the ground prepared (indicated with a check mark in parentheses); this holds, for example, for deploying safety mechanisms (Recommendation 9d) and creating environmental controls (Recommendation 10). 


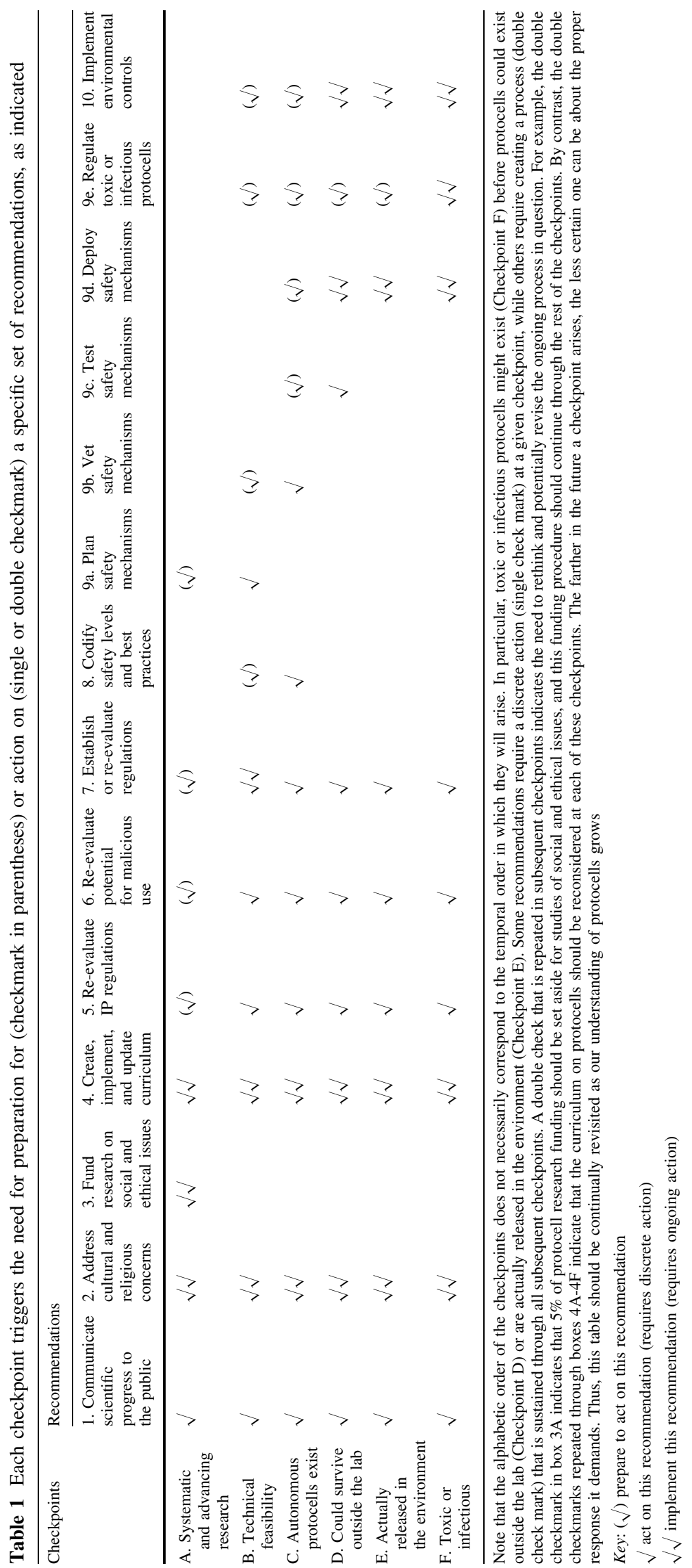




\section{Conclusions}

Protocell science and technology (Rasmussen et al. 2009a) is a bottom-up form of synthetic biology that is synthesizing novel kinds of living systems from materials that have never been alive. So, part of society's evaluation of synthetic biology in general must focus on the ethical, social, and regulatory issues that specifically involve protocell science and technology, or bottom-up synthetic biology. Many of the broader issues involving protocells have parallels in top-down synthetic biology; for example, the trajectory of development in both areas will be increasingly driven by potential commercial applications. But some broader issues are unique to protocells.

We have identified six checkpoints in protocell science and technology that call for significant ethical, social, and regulatory attention. These checkpoints are specifically relevant only for bottom-up synthetic biology. We have also proposed ten specific recommendations about the ethical, social, and regulatory aspects of protocells. The way in which specific checkpoints trigger specific recommendations is summarized in Table 1.

One of the lessons of this paper is that the details of the social and ethical implications of protocells often largely hinge on specific information that will not be available until science and technology have progressed further. So, the details of our analysis should be treated as a work in progress and continually revisited as we learn more about protocells. Nevertheless, some broad lessons can be gleaned from the overall structure of Table 1.

Table 1 vividly shows that a number of the social and ethical implications of protocells require attention right now. Although fully autonomous protocells are not yet technically feasible, the time is already ripe to communicate the state of protocell science to the general public (Recommendation 1), to address cultural and religious concerns about assembling new forms of life in the laboratory (Recommendation 2), to fund further investigation of the ethical, legal, and social implications of protocells (Recommendation 3), and to start creating a curriculum covering these issues (Recommendation 4). In addition, we should also start laying the foundation for subsequently evaluating IP regulations, potential misuse, safety mechanisms, and regulatory scope. We should stress that these recommendations are what we believe should happen, but in general they are not yet happening. So, we are sounding a call to action.

Table 1 also vividly conveys how the social and ethical implications of bottom-up and top-down synthetic biology differ. The six checkpoints for protocell research and development have no particular significance in top-down synthetic biology. The social and ethical implications of bottom-up synthetic biology unfold over time in connection with specific scientific and technical achievements in a distinctive way, summarized in Table 1. This directly results from the fact that protocells are produced in a bottom-up fashion, rather than starting with existing forms of life and then modifying them.

What animates most of the social and ethical issues concerning protocells is that protocells are for all intents and purposes autonomous forms of life. This is what raises many of the social and religious concerns; this is what makes safety mechanisms so vital; this is what drives the need for thinking through their environmental consequences; and this is what makes the ultimate impact of protocells so hard to predict.

Acknowledgments For valuable discussion of the material presented in this paper we thank our colleagues in the Programmable Artificial Cell Evolution (PACE) integrated project, EC-FP6-ISTFET-IP-002035, which supported our work, some of which is available on the PACE website. For support of this work, MB also thanks the EU project TARPOL (Targeting environmental pollution with engineered microbial systems à la carte), FP7 212894, and the Initiative for Science, Society, and Policy at the University of Southern Denmark. For helpful comments on previous versions of this manuscript, we thank Hugo Bedau, Constance Putnam, and three anonymous referees.

Open Access This article is distributed under the terms of the Creative Commons Attribution Noncommercial License which permits any noncommercial use, distribution, and reproduction in any medium, provided the original author(s) and source are credited.

\section{References}

Andrianantoandro E, Basu S, Karig DK, Weiss R (2006) Synthetic biology: new engineering rules for an emerging discipline. Mol Syst Biol 2:0028

Balmer A, Martin P (2008) Synthetic biology: social and ethical challenges. Institute for Science and Society, University of Nottingham. http://www.bbsrc.ac.uk/organisation/policies/ reviews/scientific_areas/0806_synthetic_biology.pdf\#search= $\% 22$ Synthetic\%22. Accessed May 2009

Bedau MA, Parke EC (2009a) Social and ethical issues concerning protocells. In: Rasmussen S, Bedau MA, Chen L, Deamer D, Krakauer DC, Packard NH, Stadler PF (eds) Protocells: bridging nonliving and living matter. MIT Press, Cambridge, pp 641-654

Bedau MA, Parke EC (eds) (2009b) The ethics of protocells: moral and social implications of creating life in the laboratory. MIT Press, Cambridge

BioBricks Foundation website (2009) http://bbf.openwetware.org. Accessed May 2009

Boldt J, Müller O (2008) Newtons of the leaves of grass. Nat Biotechnol 26:387-389

Brent R (2006) In the valley of the shadow of death. Draft for NAS committee. http://dspace.mit.edu/handle/1721.1/34914. Accessed May 2009

Cho MK, Magnus D, Caplan AL, McGee D (1999) Ethical considerations in synthesizing a minimal genome. Science 286:2087-2090

Cranor C (2009) The acceptability of the risks of protocells. In: Bedau MA, Parke EC (eds) The ethics of protocells: moral and social 
implications of creating life in the laboratory. MIT Press, Cambridge

Department of the Army (USA) (2008) Army special operations forces unconventional warfare FM 3-05.130. http://www.fas.org/ irp/doddir/army/fm3-05-130.pdf. Accessed May 2009

Endy D (2005) Foundations for engineering biology. Nature 438: 449-453

ETC Group (2007) Extreme genetic engineering: an introduction to synthetic biology. http://www.etcgroup.org/en/materials/publica tions.html?pub_id=602. Accessed May 2008

European Commission (2005) Synthetic biology: Applying engineering to biology. ftp://ftp.cordis.europa.eu/pub/nest/docs/synthetic biology_b5_eur21796_en.pdf. Accessed May 2009

Fukuyama F (2002) Our posthuman future: consequences of the biotechnology revolution. Farrar, Straus and Giroux

Gaisser S, Teiss T, Lunes A, Müller K, Bernauer H (2008) TESSY achievements and future perspectives in synthetic biology. http:// www.tessy-europe.eu/public_docs/TESSY-Final-Report_D5-3.pdf. Accessed May 2009

Garfinkel MS, Endy D, Epstein GL, Friedman RM (2007) Synthetic genomics: Options for governance. Final report of project funded by the Sloan Foundation. http://www.jcvi.org/cms/fileadmin/site/ research/projects/synthetic-genomics-report/synthetic-genomicsreport.pdf. Accessed May 2008

Gibson DG, Benders GA, Andrews-Pfankoch C, Denisova EA, Baden-Tillson H, Zaveri J, Stockwell TB, Brownley A, Thomas DW, Algire MA, Merryman C, Young L, Noskov VN, Glass JI, Venter JC, Hutchison CA, Smith HO (2008) Complete chemical synthesis, assembly, and cloning of a Mycoplasma genitalium genome. Science 319:1215-1220

International Human Genome Sequencing Consortium (2001) Initial sequencing and analysis of the humane genome. Nature 409:860-921

Kass L (2002) Life, liberty and the defense of dignity: the challenge for bioethics. San Francisco, Encounter Books

Kim J, White KS, Winfree E (2006) Construction of an in vitro bistable circuit from synthetic transcriptional switches. Mol Syst Biol 2:68

Lartigue C, Glass JI, Alperovich N, Pieper R, Parmar PP, Hutchison CA, Smith HO, Venter JC (2007) Genome transplantation in bacteria: changing one species into another. Science 317:632-638

Levskaya A, Chevalier AA, Tabor JJ, Simpson ZB, Lavery LA, Levy M, Davidson EA, Scourast A, Ellington AD, Marcotte EM, Voight CA (2005) Engineering Escherichia coli to see light. Nature 438:441-442

Lincoln TA, Joyce GF (2009) Self-sustained replication of an RNA enzyme. Science 323:1229-1232

Luisi PL (2002) Toward the engineering of minimal living cells. Anat Rec 268:208-214

Martin VJJ, Pitera DJ, Withers ST, Newman JD, Keasling JD (2003) Engineering a mevalonate pathway in Escherichia coli for production of terpenoids. Nat Biotechnol 21:796-802

Maurer SM, Lucas KV, Terrell S (2006) From understanding to action: Community-based options for improving safety and security in synthetic biology. UC Berkeley School of Public Policy. Draft 1.1, 15 April
O’Malley MA, Powell A, Davies JF, Calvert J (2008) Knowledgemaking distinctions in synthetic biology. Bioessays 30:57-65

Orive G, Hernández RM, Gascón AR, Calafiore R, Chang TMS, De Vos P, Hortelano G, Hunkeler D, Lacík I, Shapiro AMJ, Pedrax JL (2003) Cell encapsulation: promise and progress. Nat Med 9:104-107

PACE (Programmable Artificial Cell Evolution) website (2009) http://www.istpace.org. Accessed May 2009

Pottage A (2009) Protocell patents: property between modularity and emergence. In: Bedau MA, Parke EC (eds) The ethics of protocells: moral and social implications of creating life in the laboratory. MIT Press, Cambridge

Pullella P (2008) Pope says some science shatters human dignity. Reuters, 31 Jan 2008

Purnick PEM, Weiss R (2009) The second wave of synthetic biology: from modules to systems. Nat Rev Mol Cell Biol 10:410-422

Rasmussen S, Bedau MA, Chen L, Deamer D, Krakauer DC, Packard NH, Stadler PF (eds) (2009a) Protocells: bridging nonliving and living matter. MIT Press, Cambridge

Rasmussen S, Bedau MA, McCaskill JS, Packard NH (2009b) A roadmap to protocells. In: Rasmussen S, Bedau MA, Chen L, Deamer D, Krakauer DC, Packard NH, Stadler PF (eds) Protocells: bridging nonliving and living matter. MIT Press, Cambridge, pp 71-100

Ro D-K, Paradise EM, Ouellet M, Fisher KJ, Newman KL, Ndungu JM, Ho KA, Eachus RA, Ham TS, Kirby J, Chang MCY, Withers ST, Shiba Y, Sarpong R, Keasling JD (2006) Production of the antimalarial drug percursor artemisinic acid in engineered yeast. Nature 440:940-943

Rugh WJ (1981/2002) Nonlinear systems theory: the Volterra/Wiener approach. Originally published in 1981 by The Johns Hopkins University Press. Published on the web in 2002 at http://www. ece.jhu.edu/ rugh/214/me/volterrabook.pdf. Accessed May 2008

Schmidt M, Kelle A, Ganguli A, de Vriend H (2009) Synthetic biology: the technoscience and its societal consequences. Springer, Heidelberg

Serrano L (2007) Synthetic biology: promises and challenges. Mol Syst Biol 3:158

Shinji T (2005) Developmental trend of artificial blood (artificial red blood cells). Jpn Med Assoc J 48:135-139

Simpson ML (2006) Cell-free synthetic biology: a bottom-up approach to discovery by design. Mol Syst Biol 2:69

Smith HO, Hutchison CA, Pfannkoch C, Venter JC (2003) Generating a synthetic genome by whole genome assembly: $\phi \times 174$ bacteriophage from synthetic oligonucleotides. Proc Nat Acad Sci USA 100:15440-15445

Szostak JW, Bartel DP, Luisi PL (2001) Synthesizing life. Nature 409:387-390

USPTO (2007) Patent application 20070122826: Minimal bacterial genome. Published 31 May 2007

Venter JC et al (2001) The sequence of the human genome. Science 291:1304-1351

Zhang DY, Tuberfield AJ, Yurke B, Winfree E (2007) Engineering entropy-driven reactions and networks catalyzed by DNA. Science 318:1121-1125 